\title{
openheart Functional, morphological and electrocardiographical abnormalities in patients with apical hypertrophic cardiomyopathy and apical aneurysm: correlation with cardiac MR
}

\author{
Kenichiro Suwa, ${ }^{1}$ Hiroshi Satoh, ${ }^{1,2}$ Makoto Sano, ${ }^{1}$ Mamoru Nobuhara, ${ }^{1}$ \\ Takeji Saitoh, ${ }^{1}$ Masao Saotome,${ }^{1}$ Tsuyoshi Urushida ${ }^{1}$ Hideki Katoh, ${ }^{1}$ \\ Kei Tawarahara, ${ }^{1}$ Hayato Ohtani, ${ }^{1}$ Yasushi Wakabayashi, ${ }^{1}$ Hiroyuki Takase, ${ }^{1}$ \\ Hajime Terada, ${ }^{1}$ Yasuo Takehara, ${ }^{3}$ Harumi Sakahara, ${ }^{3}$ Hideharu Hayashi ${ }^{1}$
}

To cite: Suwa K, Satoh $\mathrm{H}$, Sano M, et al. Functional, morphological and electrocardiographical abnormalities in patients with apical hypertrophic cardiomyopathy and apical aneurysm: correlation with cardiac MR. Open Heart 2014:1:e000124. doi:10.1136/openhrt-2014000124

- Additional material is available. To view please visit the journal (http://dx.doi.org/ 10.1136/openhrt-2014000124).

Received 17 March 2014 Revised 25 June 2014 Accepted 15 July 2014

\section{CrossMark}

For numbered affiliations see end of article.

Correspondence to

Dr Hiroshi Satoh;

satoh36@hama-med.ac.jp

\section{ABSTRACT}

Objective: The prognosis of apical hypertrophic cardiomyopathy (APH) has been benign, but apical myocardial injury has prognostic importance. We studied functional, morphological and electrocardiographical abnormalities in patients with APH and with apical aneurysm and sought to find parameters that relate to apical myocardial injury.

Methods: Study design: a multicentre trans-sectional study. Patients: 45 patients with APH and 5 with apical aneurysm diagnosed with transthoracic echocardiography (TTE) in the database of Hamamatsu Circulation Forum. Measure: the apical contraction with cine-cardiac MR (CMR), the myocardial fibrotic scar with late gadolinium enhancement (LGE)-CMR, and QRS fragmentation (fQRS) defined when two ECGleads exhibited RSR's patterns.

Results: Cine-CMR revealed 27 patients with normal, 12 with hypokinetic and 11 with dyskinetic apical contraction. TTE misdiagnosed 11 (48\%) patients with hypokinetic and dyskinetic contraction as those with normal contraction. Apical LGE was apparent in 10 $(83 \%)$ and $11(100 \%)$ patients with hypokinetic and dyskinetic contraction, whereas only in 11 patients $(41 \%)$ with normal contraction $(p<0.01)$. Patients with dyskinetic apical contraction had the lowest left ventricular ejection fraction, the highest prevalence of ventricular tachycardia, and the smallest ST depression and depth of negative T waves. The presence of fQRS was associated with impaired apical contraction and apical LGE $(0 R=8.32$ and $8.61, p<0.05)$.

Conclusions: CMR is superior to TTE for analysing abnormalities of the apex in patients with APH and with apical aneurysm. The presence of fQRS can be a promising parameter for the early detection of apical myocardial injury.

\section{INTRODUCTION}

Apical hypertrophic cardiomyopathy (APH) is an uncommon phenotype of hypertrophic

\section{KEY MESSAGES}

What is already known about this subject?

- Apical injury in hypertrophic cardiomyopathy (HCM) has poor outcome. Cardiac MRI can detect regional myocardial hypertrophy not readily recognised with echocardiography in patients with HCM.

What does this study add?

- Cardiac MRI is superior to echocardiography for detection of functional and morphological abnormalities of the left ventricular apex in patients with apical hypertrophy and with apical aneurysm. The presence of fragmented QRS can help to detect apical myocardial injury.

How might this impact on clinical practice?

- To precisely estimate apical morphology and function and for early detection of apical myocardial injury in HCM.

- To apply fragmented QRS as an early indicator of apical injury in HCM.

cardiomyopathy (HCM). ${ }^{12}$ The incidence of APH is only $1-2 \%$ of HCM in Western countries, but is reported to be up to $25 \%$ in Japan. ${ }^{13}$

The prognosis of APH seems to be benign, but apical myocardial injury such as apical aneurysm and fibrosis has been related to adverse cardiac events. ${ }^{3-5}$ Although the detailed mechanisms for the development of apical aneurysm remain unknown, it is crucial to precisely analyse the functional and histological features of the left ventricular (LV) apex in patients with HCM.

Two-dimensional transthoracic echocardiography (TTE) is still the standard for the 
diagnosis of HCM, but TTE has limitations for evaluating morphology and function of the LV apex. Cardiac MRI (CMR) is now established to assess cardiac function with a high spatial resolution of cine-CMR, and to differentiate fibrosis from normal myocardium with late gadolinium enhancement (LGE)-CMR. ${ }^{6}$ Many studies have elucidated the diagnostic and prognostic values of CMR in $\mathrm{HCM},{ }^{7-12}$ but few reports showed the clinical relevance in APH and apical aneurysm. ${ }^{4} 713$

Since CMR is not necessarily available in all institutes and for all patients, and has a problem of cost, it is also necessary to predict apical myocardial injury with other imaging modalities including a 12-lead ECG. Giant negative $\mathrm{T}$ waves (GNT) with ST segment depression have been recognised as an index of apical myocardial injury and APH. ${ }^{1} 21415$ Several recent studies have suggested that the region of a myocardial scar is associated with a fragmentation of QRS complexes (fQRS) that can be a marker of a prior myocardial infarction with a substantially higher sensitivity compared with the $Q$ waves. ${ }^{16} 17$

For the precise estimation of apical morphology and function and for early detection of apical myocardial injury in HCM, we investigated apical contraction and LGE in patients with APH and with apical aneurysm using CMR, and examined the association with clinical, TTE and ECG features.

\section{PATIENTS AND METHODS Patients}

This was a multicentre trans-sectional study, and comprised 48 patients consecutively with APH and 5 with apical aneurysm who underwent CMR and were registered to the database of Hamamatsu Circulation Forum between 2004 and 2012. Patients were initially suspected to have APH because of typical ECG abnormalities with or without LV hypertrophy in other LV segments. Apical morphologies were basically diagnosed using TTE by a cardiologist or cardiac sonographer in each institute. APH was determined when apical wall thickness $>15 \mathrm{~mm}$, or ratio of apical/basal wall thickness $>1.3{ }^{13}$ Apical aneurysm was defined as a discrete thin-walled dyskinetic or akinetic segment of the most distal portion of the LV chamber. ${ }^{4}$

Patients with uncontrollable hypertension and with severe valvular diseases were not registered to the database. Obstructive atherosclerotic coronary artery disease was excluded by the absence of significant coronary arterial narrowing ( $>50 \%$ stenosis) at coronary cine or computed tomographic angiography $(\mathrm{n}=14$ for APH and $\mathrm{n}=4$ for apical aneurysm), or in the remaining patients, by absent histories of chest pain, coronary risk factors and acute coronary syndrome. All patients underwent CMR within 1 month after diagnosis with TTE.

Three patients with complete bundle branch blocks (BBBs) were then excluded from the study in consideration of modification of apical wall motion. Finally, this study consisted of 50 patients (45 patients with APH and
5 with apical aneurysm), and the database was analysed retrospectively. This study protocol was conducted in accordance with the Declaration of Helsinki.

\section{Protocol for CMR}

CMR was performed on 1.5 tesla (T) MR systems (Enshu Hospital: Excelart Vantage 1.5 T, Toshiba, Japan; Hamamatsu University Hospital: Signa Infinity Twinspeed, GE Medical Systems, Waukesha, USA; Hamamatsu Red Cross Hospital: Acheiva 1.5 T, Phillips Inc, Bothell, USA; Kosai General Hospital: Vantage Titan, Toshiba, Japan; and Seirei Mikatahara General Hospital: Signa Horizon Release 5.7, GE Medical Systems). The details of MR systems are described elsewhere. ${ }^{10} 18$ Typically, two-dimensional (2D) FIESTA and LGE images were acquired in the short axis, vertical long axis and horizontal long axis orientations. The slice thickness/gap was typically $10 \mathrm{~mm} / 0 \mathrm{~mm}$ (6-9 slices).

Breath-hold cine MRIs were obtained in contiguous short-axis planes from apex to base of the heart with the patient in a resting state. The 2D FIESTA cine images were based on the steady state free precession sequence. The imaging parameters were as follows: matrix of $192 \times 192$, field of view of $34 \mathrm{~cm}$, flip angle of $45^{\circ}$ and readout bandwidth of $125 \mathrm{kHz}$. Sixteen data lines were acquired per segment.

LGE images were acquired $15 \mathrm{~min}$ after an injection of $0.2 \mathrm{mmol} / \mathrm{kg}$ of contrast material (Gd-DTPA-BMA, Fuji Pharma, Tokyo, Japan). LGE imaging was based on the inversion recovery prepared fast gradient echo (IR-FGRE) sequence. The imaging parameters were as follows: matrix of $256 \times 160$, field of view of $34 \mathrm{~cm}$, flip angle of $20^{\circ}$ and readout bandwidth of $31.25 \mathrm{kHz}$. The IR-FGRE technique was repeated during every R-to-R interval and the trigger delay was $300 \mathrm{~ms}$. The readout data line was 160 each, where 24 data lines were acquired per segment. The optimum inversion time (200-240 ms) was measured right before the LGE imaging.

\section{Analyses of CMR}

Two experienced cardiovascular radiologists (KS and HS) interpreted all the CMRs without knowledge of clinical findings. LV end-diastolic volume (LVEDV), endsystolic volume (LVESV), left ventricular ejection fraction (LVEF) and LV mass (LVM) were acquired from the 2D FIESTA cine images in short axis view. The values for $\mathrm{LV}$ volume and mass were indexed by dividing them with body surface area (LVEDV index, LVESV index and LVM index).

We classified patients into three groups according to apical contraction: (1) normal: apical hypertrophy and spade-like morphology at end-diastole and a disappearance of apical cavity at end-systole, (2) hypokinetic: apical hypertrophy and spade-like morphology at enddiastole with retention of apical cavity at end-systole and (3) dyskinetic: apical wall thinning and dyskinetic apical contraction (with or without ventricular obstruction; 

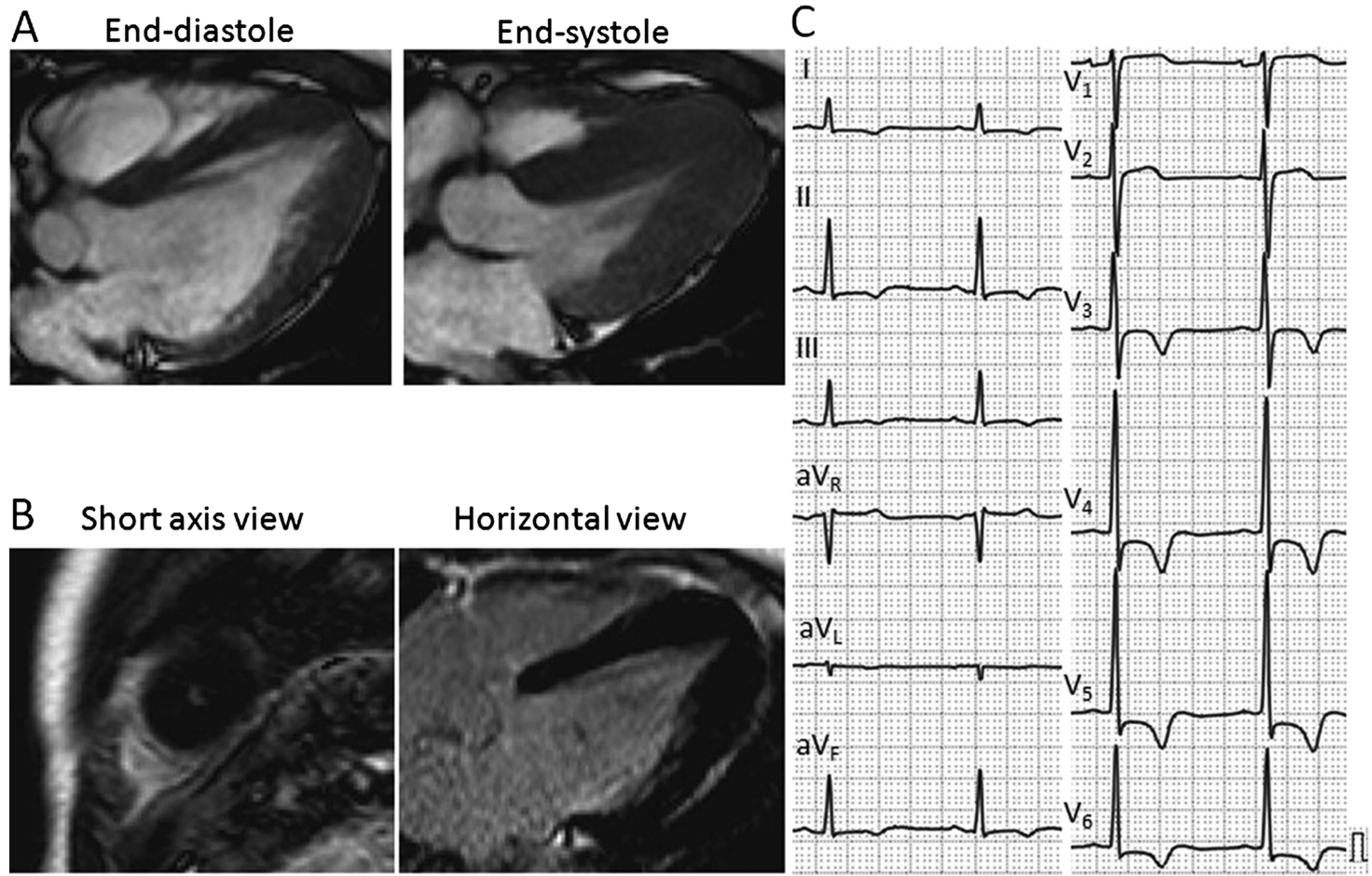

Figure 1 A representative patient with normal apical contraction. (A) Cine-cardiac MRIs (CMRs) at end-diastole (left) and end-systole (right). Apical hypertrophy and spade-like morphology at end-diastole and a complete disappearance of apical cavity at end-systole were shown. (B) Late gadolinium enhancement (LGE)-CMRs at short-axis view (left) and horizontal long-axis view (right). No LGE was observed. (C) Standard 12-lead ECG. High voltage QRS complexes, strain type ST depression and giant negative $\mathrm{T}$ waves were apparent.

figures 1-3). Regional analyses of LGE-CMR were performed using the 17 -segment model. ${ }^{15}$ Then we examined the presence of LGE in each segment, determined the presence or absence of apical LGE and counted the number of segments with LGE. The apical injury was defined to be impaired apical contraction (hypokinetic or dyskinetic contraction) or the presence of apical LGE. The apical contraction and the presence, location and extent of LGE were determined by the consensus of the two observers with analyses of all the orientations.

\section{Analyses of 12-lead ECG and TTE}

Twelve-lead ECG and TTE were performed before CMR $(<1$ month $)$ in all patients. We examined the presence of fQRS, which was defined when notched $\mathrm{R}$ or $\mathrm{S}$ were present in at least two-ECG leads (figure 2 and 3). ${ }^{16}$ The apical motion was analysed with 2D mode of TTE (apical 2 and 4 chamber views) by a cardiologist in each institute. Patients also underwent a $24 \mathrm{~h}$ ambulatory Holter ECG. The intervals between the analyses by CMR and Holter ECG were <3 months. Non-sustained ventricular tachycardia (VT) and supraventricular tachycardia (SVT) were defined as three or more consecutive premature complexes with a heart rate of $>100 \mathrm{bpm}$.

\section{Statistical analysis}

All data were expressed as the means $\pm S D$ of the indicated numbers (n) or percentages, as appropriate.
Categorical variables were compared among the groups by $\chi^{2}$ analysis. Continuous variables were compared by unpaired $t$ test or one-way analysis of variance followed by Scheffe's post hoc analysis. Differences were considered to be significant when $\mathrm{p}<0.05$. In the multivariate analysis, only ECG or TTE parameters that showed $p<0.1$ in the univariate analyses were incorporated. The sensitivity and specificity of fQRS for impaired apical contraction and apical LGE were derived from dividing the patient numbers with or without fQRS from those with or without impaired apical contraction or apical LGE. All the statistical analyses were performed using software StatFlex (V.6).

\section{RESULTS \\ Estimation of apical contraction}

All patients were assigned to three groups according to apical contraction estimated with cine-CMR: normal $(\mathrm{n}=27)$, hypokinetic $(\mathrm{n}=12)$ and dyskinetic $(\mathrm{n}=11)$ apical contraction, respectively. TTE underestimated $6(50 \%)$ and $5(45 \%)$ patients with hypokinetic and dyskinetic apical contraction in cine-CMR as those with normal apical contraction, and also $1(9 \%)$ patient with dyskinetic apical contraction as hypokinetic apical contraction. Additionally, TTE overestimated $2(7 \%)$ patients with normal apical contraction as those with hypokinetic apical contraction, and $1(8 \%)$ patient with hypokinetic apical contraction as dyskinetic apical contraction (see 

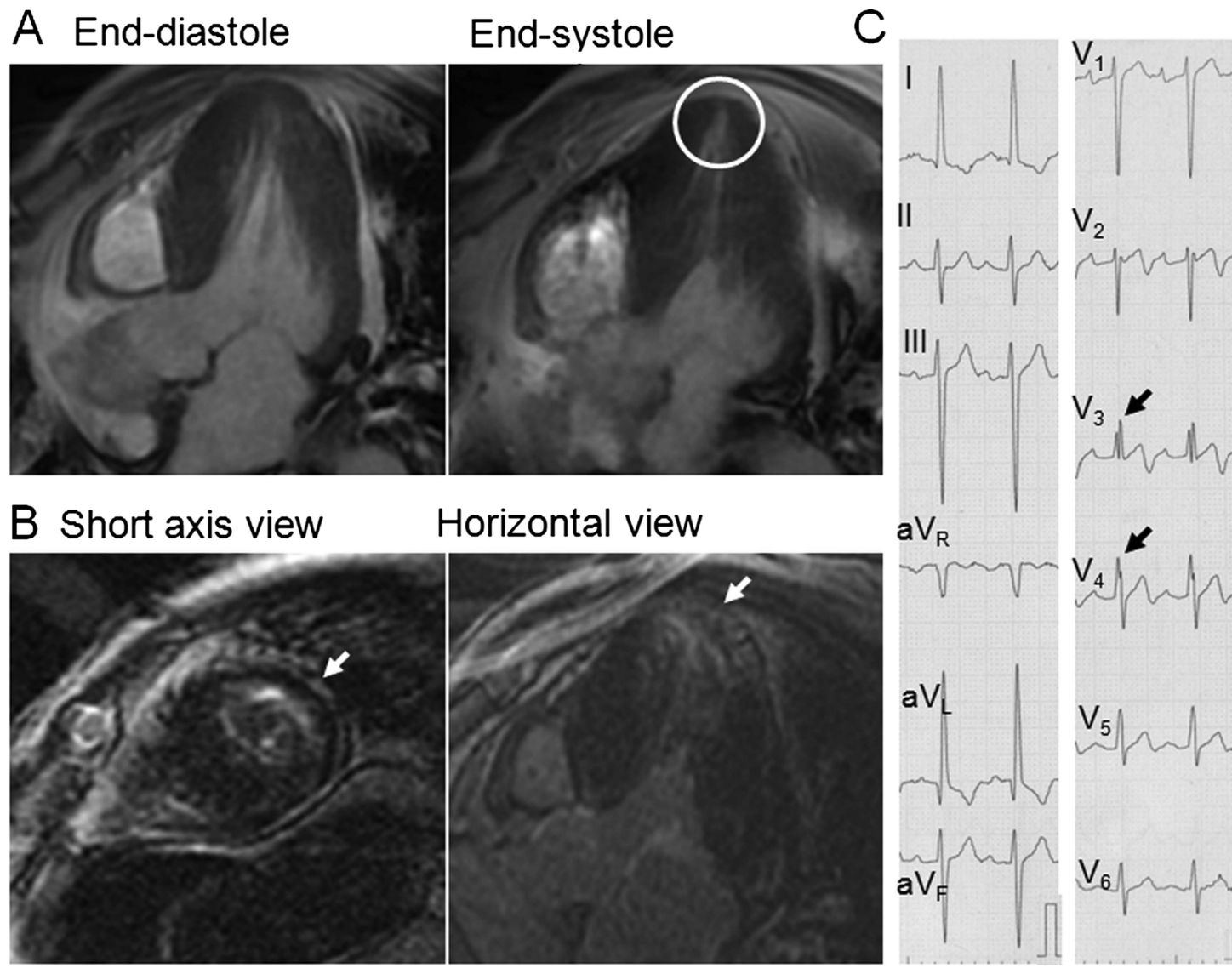

Horizontal view
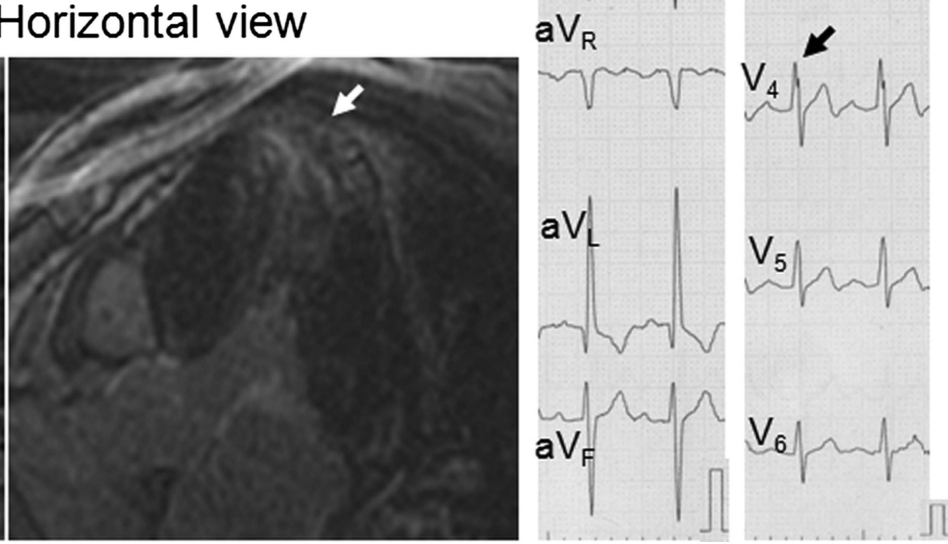

Figure 2 A representative patient with hypokinetic apical contraction. (A) Cine-cardiac MRIs (CMRs) at end-diastole (left) and end-systole (right). Apical hypertrophy and spade-like morphology at end-diastole were similar to those in a patient with normal apical contraction, but the apical cavity retained and did not completely disappear at end-systole (white circle). (B) Late gadolinium enhancement (LGE)-CMRs at short-axis view (left) and horizontal long-axis view (right). LGE at the left ventricular apex was apparent (white arrows). (C) Standard 12-lead ECG. In addition to giant negative T waves, fragmented QRS complexes at $\mathrm{V}_{3}$ and $\mathrm{V}_{4}$ leads were apparent (black arrows).

online supplementary table S1). There was no tendency in the prevalence of misdiagnosis among the institutes or analysts. Apical thrombus was detected in one patient with dyskinetic apical contraction by TTE and CMR.

\section{Representative images}

Figures 1-3 show representative cine-CMRs and LGE-CMRs, and 12-lead ECGs in patients with normal, hypokinetic and dyskinetic apical contraction. LGE-CMRs exhibited LGE at the LV apex in patients with hypokinetic and with dyskinetic apical contraction, but did not in the patient with normal apical contraction. The 12-lead ECGs demonstrated high voltage QRS complexes, strain type ST depression and GNT in the patients, although the degree of ST depression and the depth of GNT were to a lesser extent in patients with hypokinetic and with dyskinetic apical contraction. Contrarily, fQRS was present in only patients with hypokinetic and with dyskinetic apical contraction.

\section{CIMR findings}

Table 1 demonstrates the differences in CMR parameters among the three patient groups. LVEF was significantly lower in patients with dyskinetic apical contraction than those with normal or hypokinetic apical contraction, whereas LV volume, mass or maximum segmental wall thickness did not differ. LGE in the LV apex was found in all patient groups, but the prevalence was relatively low in patients with normal apical contraction $(41 \%)$, whereas most patients with hypokinetic apical contraction $(83 \%)$ and all patients with dyskinetic apical contraction (100\%) had LGE in the LV apex. There was no difference in the number of LGE segments among the groups.

\section{Clinical features}

We then compared clinical features, TTE and ECG parameters among patients with different apical contraction and between patients with and without apical LGE (tables 2-4 and see online supplementary tables S2-4). The prevalence of VT and medication with $\beta$-blockers were highest in patients with dyskinetic apical contraction. The prevalence of VT, SVT, and medication with antiarrhythmic agents and antithrombotic agents was higher in patients with apical LGE. No patients had signs of systemic thromboembolism. Age, sex, symptoms, 
A End-diastole

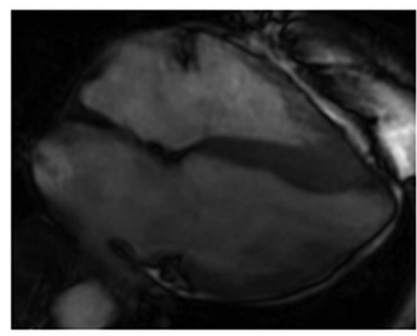

B Short axis view

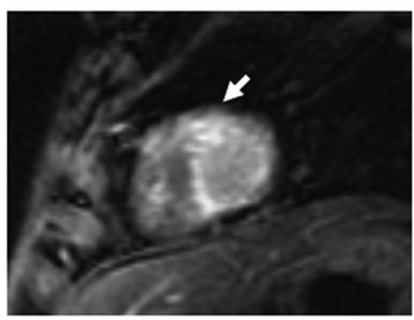

End-systole
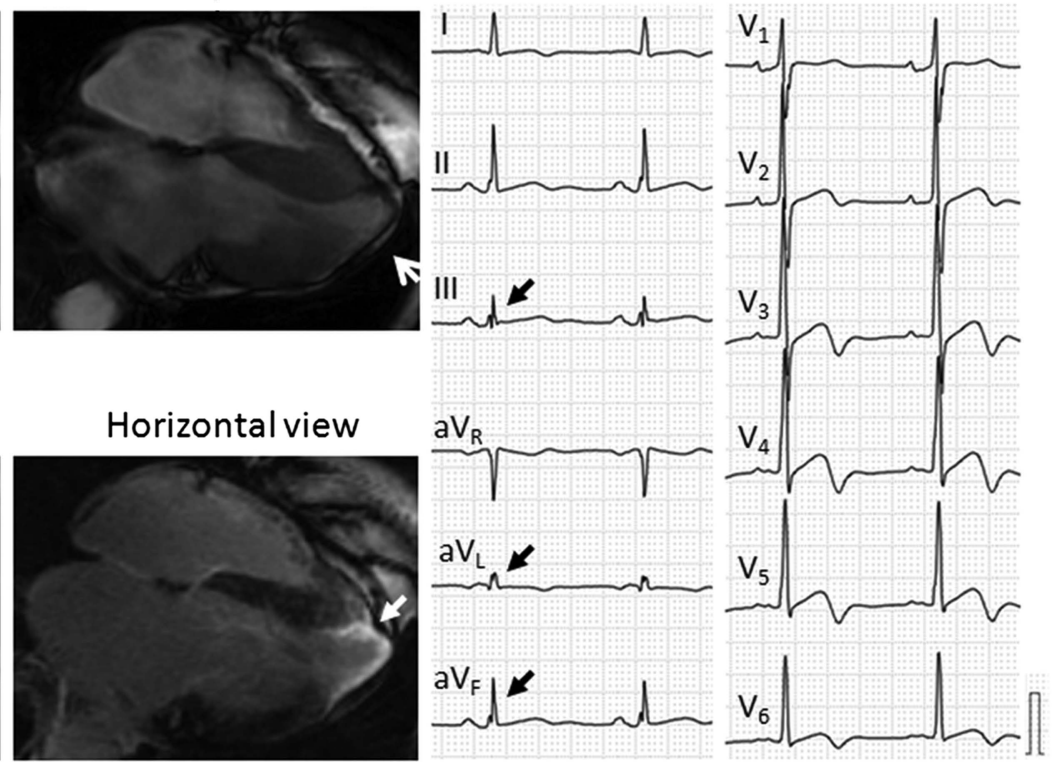

Figure 3 A representative patient with dyskinetic apical contraction. (A) Cine-cardiac MRIs (CMRs) at end-diastole (left) and end-systole (right). The apical wall thinning, dyskinetic apical contraction and apical pouch were apparent. (B) Late gadolinium enhancement (LGE)-CMR images at short-axis view (left) and horizontal long axis view (right). LGE at the left ventricular apex was apparent (white arrows). (C) Standard 12-lead ECG. In addition to high voltage QRS complexes and giant negative T waves, fragmented $\mathrm{QRS}$ complexes at III, $\mathrm{aV}_{\mathrm{L}}$ and $\mathrm{aV} \mathrm{F}_{\mathrm{F}}$ leads were apparent (black arrows).

family history and serum NT-proBNP level did not significantly differ.

TTE parameters did not significantly differ among patients with different apical contraction or between patients with and without apical LGE. In 12-lead ECG, the maximum ST depression and maximum depth of GNT were smaller in patients with dyskinetic apical contraction than those in the other two groups. The prevalence of fQRS was higher in patients with hypokinetic or with dyskinetic apical contraction than that in patients with normal apical contraction. The smaller maximum ST depression and higher prevalence of fQRS were also found in patients with apical LGE. fQRS was present dominantly at inferior leads (III, $\mathrm{aV}_{\mathrm{F}}$ ) and mid-precordial leads $\left(\mathrm{V}_{3}, \mathrm{~V}_{4}\right.$; see online supplementary table S5). Patients with fQRS had a higher prevalence of VT than those without fQRS ( $38 \%$ vs $14 \%, \mathrm{p}<0.05)$.

\section{Multivariate analyses for apical contraction and apical LGE}

The multivariate analyses showed that fQRS was the sole index of impaired apical contraction (hypokinetic and dyskinetic apical contraction) and apical LGE (table 5), but was not an index of dyskinetic apical contraction

Table 1 CMR parameters in patients with normal, hypokinetic and dyskinetic apical contraction

\begin{tabular}{|c|c|c|c|c|}
\hline $\begin{array}{l}\text { Apical contraction } \\
\text { Number }\end{array}$ & $\begin{array}{l}\text { Normal } \\
27\end{array}$ & $\begin{array}{l}\text { Hypokinetic } \\
12\end{array}$ & $\begin{array}{l}\text { Dyskinetic } \\
11\end{array}$ & p Value \\
\hline \multicolumn{5}{|l|}{ Cine-CMR } \\
\hline LVEF (\%) & $66.2 \pm 12.3$ & $65.9 \pm 7.61$ & $56.4 \pm 10.5^{\star}$ & 0.04 \\
\hline LVMI $\left(\mathrm{g} / \mathrm{m}^{2}\right)$ & $63.7 \pm 26.0$ & $64.6 \pm 25.4$ & $87.4 \pm 25.9$ & 0.05 \\
\hline LVEDVI $\left(\mathrm{mL} / \mathrm{m}^{2}\right)$ & $60.4 \pm 16.3$ & $57.7 \pm 19.0$ & $66.2 \pm 12.3$ & 0.44 \\
\hline LVESVI $\left(\mathrm{mL} / \mathrm{m}^{2}\right)$ & $20.0 \pm 9.6$ & $20.7 \pm 9.5$ & $27.4 \pm 7.1$ & 0.08 \\
\hline Maximum LVWT (mm) & $18.3 \pm 4.2$ & $19.2 \pm 5.8$ & $20.3 \pm 3.8$ & 0.48 \\
\hline \multicolumn{5}{|l|}{ LGE-CMR } \\
\hline LGE in any LV wall & $16(59.3 \%)$ & $9(75.0 \%)$ & $11(100 \%)$ & 0.04 \\
\hline In the apex & $11(40.7 \%)$ & $10(83.3 \%)$ & $11(100 \%)$ & $<0.001$ \\
\hline In other LV segments & $11(40.7 \%)$ & $7(58.3 \%)$ & $9(81.8 \%)$ & 0.06 \\
\hline Number of LGE segments & $3.5 \pm 4.9$ & $2.8 \pm 2.7$ & $7.0 \pm 4.2$ & 0.05 \\
\hline
\end{tabular}


Table 2 Comparison of clinical features in terms of apical contraction

\begin{tabular}{|c|c|c|c|c|}
\hline Number & $\begin{array}{l}\text { Normal } \\
27\end{array}$ & $\begin{array}{l}\text { Hypokinetic } \\
12\end{array}$ & $\begin{array}{l}\text { Dyskinetic } \\
11\end{array}$ & p Value \\
\hline Age (years) & $66.0 \pm 9.84$ & $62.4 \pm 11.8$ & $65.5 \pm 14.1$ & 0.64 \\
\hline Male & $21(77.8 \%)$ & $10(83.3 \%)$ & $11(100 \%)$ & 0.24 \\
\hline \multicolumn{5}{|l|}{ Symptom } \\
\hline Chest pain & $11(40.7 \%)$ & $3(25.0 \%)$ & 4 (36.4\%) & 0.64 \\
\hline Palpitation & $10(37.0 \%)$ & 4 (33.3\%) & $2(18.2 \%)$ & 0.52 \\
\hline Syncope & $2(7.4 \%)$ & $2(16.7 \%)$ & $2(18.2 \%)$ & 0.55 \\
\hline \multicolumn{5}{|l|}{ NYHA } \\
\hline I & $22(81.5 \%)$ & $9(75.0 \%)$ & 8 (72.7\%) & 0.81 \\
\hline II & 5 (18.5\%) & $3(25.0 \%)$ & $3(27.3 \%)$ & 0.81 \\
\hline III/IV & $0(0 \%)$ & $0(0 \%)$ & $0(0 \%)$ & \\
\hline \multicolumn{5}{|l|}{ Family history } \\
\hline Sudden death & $0(0 \%)$ & $0(0 \%)$ & $1(9.1 \%)$ & 0.16 \\
\hline $\mathrm{HCM}$ & $3(11.1 \%)$ & $0(0 \%)$ & $1(9.1 \%)$ & 0.49 \\
\hline \multicolumn{5}{|l|}{ Past history } \\
\hline VT & $4(14.8 \%)$ & $2(16.7 \%)$ & $6(54.5 \%)$ & 0.03 \\
\hline SVT or Af & 9 (33.3\%) & $6(50.0 \%)$ & $3(27.3 \%)$ & 0.48 \\
\hline Stroke & $2(7.4 \%)$ & $1(8.3 \%)$ & $3(27.3 \%)$ & 0.21 \\
\hline NT pro-BNP (ng/mL) & $246.0(95-1252)$ & $247.0(38-603)$ & 262.5 (122-361) & 0.63 \\
\hline \multicolumn{5}{|l|}{ Medication } \\
\hline ACEI/ARB & $16(59.3 \%)$ & $9(75.0 \%)$ & $6(54.5 \%)$ & 0.55 \\
\hline Ca blockers & 9 (33.3\%) & 6 (50.0\%) & 3 (27.3\%) & 0.48 \\
\hline$\beta$-blockers & 7 (25.9\%) & 7 (58.3\%) & 7 (63.6\%) & 0.04 \\
\hline AAA & $2(7.4 \%)$ & 3 (25.0\%) & 2 (18.2\%) & 0.31 \\
\hline ATA & $4(14.8 \%)$ & 2 (16.7\%) & 4 (36.4\%) & 0.31 \\
\hline
\end{tabular}

Each value is number (\%), mean \pm SD or median (range).

AAA, antiarrhythmic agents; ACEI, ACE inhibitors; Af, atrial fibrillation; ARB, angiotensin II receptor blockers; ATA, antithrombotic agents; HCM, hypertrophic cardiomyopathy; NYHA, New York Heart Association functional class; SVT, supraventricular tachycardia; VT, ventricular tachycardia.

(data not shown). The sensitivity and specificity of fQRS were $65.2 \% \quad(15 / 23$ patients $)$ and $77.8 \% \quad(21 / 27$ patients) for impaired apical contraction, and $59.4 \%$ (19/32 patients) and $88.9 \%$ (16/18 patients) for apical LGE, respectively. The PR interval was also an index of impaired apical contraction, although it did not reach significance in the univariate analysis.

\section{DISCUSSION}

We examined apical morphology and function in patients with APH and with apical aneurysm and showed that: (1) cine-CMR could identify impaired apical contraction more precisely compared with TTE, (2) the prevalence of apical LGE was higher in patients with hypokinetic and dyskinetic apical contraction than in those with normal apical contraction, and (3) the presence of $f Q R S$ was associated with impaired apical contraction and apical LGE. We could emphasise the clinical usefulness of CMR for estimating apical morphology and function, and provide, for the first time, the relevance of $\mathrm{fQRS}$ for prediction of apical myocardial injury.

\section{APH and apical aneurysm in HCM}

APH has a benign prognosis in Japan, with the exception of elderly patients, but the condition appears to be less benign in Western countries. ${ }^{3} 519$ The prognosis of patients with HCM becomes quite poor when the apex contains aneurysms. In a report from Maron et al, ${ }^{4} 43 \%$ of patients with apical aneurysm experienced adverse cardiac events. The intraventricular pressure gradient due to mid-ventricular obstruction can trigger chronic myocardial ischaemia, which in turn results in apical aneurysm formation. ${ }^{20}$ The severe apical hypertrophy could also impose pressure overload and coronary flow impairment of the LV apex, which may thereby provoke myocardial infarction and/or fibrosis. ${ }^{21}$ All patients with dyskinetic apical contraction had apical LGE, although there was no difference in the thickness of interventricular septum or posterior wall. Patients with dyskinetic apical contraction and those with apical LGE had higher prevalence of VT. Thus, myocardial fibrosis at the LV apex was closely associated with dyskinetic apical contraction and development of VT.

\section{Estimation of apical abnormalities with TTE and CMR}

Although TTE has been the standard tool for the diagnosis of HCM, it has limitations for precise visualisation of whole ventricles and quantification of hypertrophy, especially for the LV apex. In addition to apical views, a careful approach with sequential parasternal short axis view is required. CMR can image in any plane and 
Table 3 Comparison of TTE parameters in terms of apical contraction

\begin{tabular}{|c|c|c|c|c|}
\hline Number & $\begin{array}{l}\text { Normal } \\
27\end{array}$ & $\begin{array}{l}\text { Hypokinetic } \\
12\end{array}$ & $\begin{array}{l}\text { Dyskinetic } \\
11\end{array}$ & p Value \\
\hline LVDd (mm) & $45.6 \pm 7.7$ & $47.3 \pm 6.2$ & $46.4 \pm 7.6$ & 0.79 \\
\hline LVDs (mm) & $27.2 \pm 5.6$ & $27.7 \pm 3.9$ & $29.0 \pm 4.9$ & 0.61 \\
\hline IVST (mm) & $13.8 \pm 3.4$ & $13.1 \pm 2.7$ & $11.6 \pm 3.7$ & 0.20 \\
\hline PWT (mm) & $12.1 \pm 2.5$ & $10.9 \pm 1.5$ & $10.9 \pm 2.1$ & 0.14 \\
\hline LVEF (\%) & $70.9 \pm 9.4$ & $72.3 \pm 8.0$ & $67.7 \pm 4.6$ & 0.41 \\
\hline LVFS (\%) & $40.0 \pm 7.6$ & $40.2 \pm 5.0$ & $37.7 \pm 3.8$ & 0.55 \\
\hline LAD (mm) & $37.1 \pm 6.5$ & $39.2 \pm 5.3$ & $41.2 \pm 4.9$ & 0.14 \\
\hline E/A ratio & $0.85 \pm 0.24$ & $1.07 \pm 0.43$ & $0.96 \pm 0.39$ & 0.17 \\
\hline DcT (ms) & $226.8 \pm 37.5$ & $209.5 \pm 60.7$ & $212.2 \pm 56.8$ & 0.55 \\
\hline E/e' ratio & $15.5 \pm 4.7$ & $15.5 \pm 4.7$ & $13.4 \pm 4.4$ & 0.45 \\
\hline
\end{tabular}

Each value is mean \pm SD.

DcT, deceleration time of early left ventricular inflow; $E / A$, the ratio of $E$ and $A$ waves in mitral inflow velocities; $E / e^{\prime}$, the ratio of $E$ wave and early peak of diastolic annular velocity (e'); IVST and PWT, thickness of interventricular septum and posterior wall; LAD, left atrial dimension; LVDd and LVDs, left ventricular end-diastolic and end-systolic dimensions; LVEF, left ventricular ejection fraction; LVFS, left ventricular fractional shortening; TTE, transthoracic echocardiography.

cine-CMR has achieved high spatial resolution with a substantial improvement of blood to myocardium contrast. Since Moon et $a l^{7}$ reported a series of patients in whom CMR could detect unrecognised APH, several studies have showed the usefulness of CMR for detection

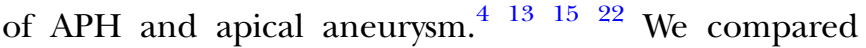
apical contraction with TTE and cine-CMR in patients who were recognised as having APH or apical aneurysm. In addition to dyskinetic apical contraction, we defined hypokinetic apical contraction when the apical cavity retained at end-systole. This estimation was quite novel, and TTE underestimated $50 \%$ and $45 \%$ patients with hypokinetic and dyskinetic apical contraction, respectively, as normal. The misdiagnosis was not ascribed to different institutes or analysts. Maron et al $t^{4}$ studied 28 of 1299 patients with HCM who had apical aneurysm, and TTE could identify apical aneurysm in only 16 patients, but CMR could detect it in the remaining 12 patients.

The high prevalence and particular distribution patterns of LGE in the LV wall further emphasise the clinical usefulness of CMR for the diagnosis of
HCM. $^{10} 152324$ Although apical aneurysms relate to adverse cardiac events, the clinical implications of hypokinetic apical contraction in APH remain undefined. In this study, most patients with hypokinetic apical contraction had LGE in the LV apex. The higher prevalence of LGE in the LV apex in patients with hypokinetic apical contraction suggests more advanced myocardial fibrosis than in those with normal apical contraction. Although there is no evidence that patients with hypokinetic apical contraction progressively develop apical aneurysm and/or adverse cardiac events, early detection of apical myocardial injury with CMR may help for risk stratification and management of APH. Patients with hypokinetic apical contraction, but without LGE, may benefit from aggressive therapies with $\beta$-adrenergic receptor blockers or inhibitors of renin-angiotensin-aldosterone systems.

There were no differences in standard TTE parameters in terms of apical contraction and apical LGE, although the mid-ventricular hypertrophy was expected to have caused a chronic intraventricular pressure gradient and, as a result, apical myocardial injury. The reason for the lack

Table 4 Comparison of ECG parameters in terms of apical contraction

\begin{tabular}{|c|c|c|c|c|}
\hline Number & $\begin{array}{l}\text { Normal } \\
27\end{array}$ & $\begin{array}{l}\text { Hypokinetic } \\
12\end{array}$ & $\begin{array}{l}\text { Dyskinetic } \\
11\end{array}$ & p Value \\
\hline HR (bpm) & $65.5 \pm 11.4$ & $65.4 \pm 16.0$ & $67.4 \pm 11.9$ & 0.92 \\
\hline $\mathrm{PR}(\mathrm{ms})$ & $163.7 \pm 26.9$ & $173.1 \pm 38.4$ & $189.6 \pm 25.4$ & 0.07 \\
\hline QRS (ms) & $100.0 \pm 15.7$ & $96.7 \pm 19.9$ & $102.2 \pm 7.07$ & 0.69 \\
\hline QT (ms) & $429 \pm 30.7$ & $438 \pm 44.2$ & $421 \pm 43.1$ & 0.55 \\
\hline QTc (ms) & $440 \pm 26.0$ & $452 \pm 15.2$ & $437 \pm 31.5$ & 0.33 \\
\hline Sokolow-Lyon voltage (mV) & $4.89 \pm 1.48$ & $4.42 \pm 1.83$ & $3.50 \pm 1.75$ & 0.07 \\
\hline $\operatorname{Max} \mathrm{ST}(\mathrm{mV})$ & $-0.14 \pm 0.18$ & $-0.18 \pm 0.14$ & $0.02 \pm 0.18^{\star \star \star}$ & 0.02 \\
\hline Max T waves (mV) & $-1.02 \pm 0.63$ & $-1.02 \pm 0.40$ & $-0.43 \pm 0.59^{*}$ & 0.02 \\
\hline fQRS & $6(22.2 \%)$ & $7(58.3 \%)$ & $8(72.7 \%)$ & 0.01 \\
\hline
\end{tabular}

Each value is mean \pm SD or number (\%). ${ }^{*} p<0.05$ vs Normal, ${ }^{* *} p<0.05$ vs hypokinetic by Scheffe's post hoc analyses after one-way analysis of variance.

fQRS, fragmented QRS complexes; HR, heart rate; Max ST, maximum level of ST change; Max T waves, maximum amplitude of positive or negative T waves at lateral precordial leads; QTc, corrected QT interval. 
Table 5 Multivariate analyses in terms of apical contraction and apical LGE

Impaired apical contraction

\begin{tabular}{llll}
\hline Variables & OR & 95\% Cl & p Value \\
\hline fQRS & 8.32 & 1.80 to 38.5 & 0.01 \\
PR & 1.03 & 1.00 to 1.06 & 0.04 \\
Sokolow-Lyon voltage & 0.74 & 0.44 to 1.24 & 0.22 \\
ST & 0.08 & 0.00 to 18.6 & 0.31 \\
T wave & 1.89 & 0.43 to 8.32 & 0.41
\end{tabular}

Apical LGE

\begin{tabular}{llll}
\hline Variables & OR & $95 \%$ Cl & p Value \\
\hline fQRS & 8.61 & 1.51 to 49.1 & 0.02 \\
PR & 1.01 & 0.99 to 1.04 & 0.33 \\
QRS & 1.04 & 0.98 to 1.10 & 0.19 \\
ST & 26.7 & 0.13 to 5322 & 0.22 \\
\hline
\end{tabular}

fQRS, fragmented QRS complexes; LGE, late gadolinium enhancement.

of differences remains unknown, but may be dependent on heterogeneous LV hypertrophy in such patients. ${ }^{22}$

\section{Prediction of apical myocardial injury with 12-lead ECG}

In HCM, a 12-lead ECG shows a wide variety of abnormal patterns and provides diagnostic and prognostic information. We, as well as Dumont et al ${ }^{1525}$ have exhibited correlation between LGE and conduction disturbance, abnormal $\mathrm{Q}$ waves and GNT. However, studies for ECG abnormalities that relate to apical myocardial injury remain inadequate.

GNT are recognised as the most typical feature of $\mathrm{APH}$, at least in Japanese patients. ${ }^{1}{ }^{2}$ The size of GNT has been related to the apical wall thickness and asymmetric distal hypertrophy. ${ }^{14} 15$ The maximum ST depression was smaller in patients with dyskinetic apical contraction and with apical LGE than those in other groups. The maximum depth of GNT was smaller in patients with dyskinetic apical contraction, but did not differ between patients with and without apical LGE. Previous studies showed a disappearance of GNT during a long-term follow-up. ${ }^{26}{ }^{27}$ Given that the relative myocardial ischaemia caused ST-T abnormalities in patients with $\mathrm{APH},{ }^{21} 28$ the apical wall thinning and fibrosis can be mechanisms of the decreases in ST depression. However, the correlation between the depth of GNT and apical fibrosis remains controversial. ${ }^{15} 29$ The reason why PR interval became significant in the multivariate analysis for impaired apical contraction is also uncertain.

However, the most important finding was that fQRS, without an evidence of BBB, was associated with impaired apical contraction and apical LGE. Actually, fQRS at inferior and/or mid-precordial leads was apparent in several previous case reports showing apical aneurysm formation in APH. ${ }^{13} 2030$ The finding that fQRS was also related with hypokinetic apical contraction suggested that fQRS could be an index of myocardial injury before the formation of apical aneurysm.
Various prior studies have suggested that the region of a myocardial scar is associated with alteration in QRS morphology, leading to a terminal conduction delay or a fragmentation of QRS complexes. ${ }^{16}{ }^{17}$ Das et $a l^{16}$ showed that $\mathrm{fQRS}$ is a marker of a prior myocardial infarction, defined by regional perfusion abnormalities, that has a substantially higher sensitivity and negative predictive value compared with the $Q$ waves. We showed moderately high sensitivity and specificity of fQRS for detection of apical myocardial injury. Das $e t a l^{16}$ also reported that the fQRS is an independent predictor of cardiac events in patients with coronary arterial disease. In this study, fQRS was associated with a higher prevalence of VT. Despite the analysis in small patient groups and the lack of prognostic information, fQRS can be a promising parameter for the early detection of apical myocardial injury and the start of treatment in patients with APH.

\section{Limitations}

This study consisted of patients who underwent CMR at five different institutes. The selection bias, different equipment and small sample size may be limitations for extrapolating our data to diverse patient groups. Coronary angiography was not routinely performed to exclude coronary arterial disease, but a section of patients were diagnosed by a lack of symptoms or risk factors. Although there were no significant stenotic coronary lesions in patients with apical aneurysm, the possibility of coronary spasm or coronary embolism cannot be excluded. We evaluated apical contraction and apical LGE as indices of apical injury. The estimation of apical wall thickening and the quantification of apical fibrosis (possibly with T1 mapping) may provide more precise information. Finally, we did not examine the relevance of apical myocardial injury in terms of adverse cardiac events during a long-term follow-up.

\section{CONCLUSIONS}

CMR was superior to TTE for detection of functional and morphological abnormalities of the LV apex in patients with APH and with apical aneurysm. The presence of fQRS can help for suspected apical myocardial injury. More advanced diagnostic and prognostic evaluation in a larger population can clarify the clinical relevance of CMR and $\mathrm{FQRS}$ in those patients.

\section{Author affiliations}

${ }^{1}$ The Investigator Group, Hamamatsu Circulation Forum; (Hamamatsu Circulation Forum consists of Enshu Hospital, Hamamatsu University Hospital, Hamamatsu Red Cross Hospital, Kosai General Hospital and Seirei Mikatahara Hospital.)

${ }^{2}$ Division of Cardiology, Internal Medicine III, Hamamatsu University School of Medicine, Hamamatsu, Japan

${ }^{3}$ Department of Radiology, Hamamatsu University School of Medicine, Hamamatsu, Japan

Contributors KS was involved in description of manuscript. HS was involved in description and revision of manuscript. MS and MS were involved in analysis of MRI data. MN and TS were involved in analysis of echo and ECG 
data. TU, HK, HO, YW, KT, HT and HT were involved in enrolment of patients. $\mathrm{YT}$ and $\mathrm{HS}$ were involved in MRI technical support. $\mathrm{HH}$ was involved in general control of the study.

Funding This research received no specific grant from any funding agency in the public, commercial or not-for-profit sectors.

Competing interests None.

Patient consent Obtained.

Ethics approval Hamamatsu Univ Sch Med

Provenance and peer review Not commissioned; externally peer reviewed.

Data sharing statement No additional data are available.

Open Access This is an Open Access article distributed in accordance with the Creative Commons Attribution Non Commercial (CC BY-NC 4.0) license, which permits others to distribute, remix, adapt, build upon this work noncommercially, and license their derivative works on different terms, provided the original work is properly cited and the use is non-commercial. See: http:// creativecommons.org/licenses/by-nc/4.0/

\section{REFERENCES}

1. Sakamoto T, Tei C, Murayama M, et al. Giant T wave inversion as a manifestation of asymmetrical apical hypertrophy $(\mathrm{AAH})$ of the left ventricle: echocardiographic and ultrasono-cardiotomographic study. Jpn Heart J 1976;17:611-29.

2. Yamaguchi $\mathrm{H}$, Ishimura $\mathrm{T}$, Nishiyama $\mathrm{S}$, et al. Hypertrophic nonobstructive cardiomyopathy with giant negative $T$ waves (apical hypertrophy): ventriculographic and echocardiographic features in 30 patients. Am J Cardiol 1979;44:401-12.

3. Eriksson MJ, Sonnenberg B, Woo A, et al. Long-term outcome in patients with apical hypertrophic cardiomyopathy. J Am Coll Cardiol 2002;39:638-45.

4. Maron MS, Finley JJ, Bos JM, et al. Prevalence, clinical significance, and natural history of left ventricular apical aneurysms in hypertrophic cardiomyopathy. Circulation 2008;118:1541-9.

5. Chung T, Yiannikas J, Freedman SB, et al. Unusual features of apical hypertrophic cardiomyopathy. Am J Cardiol 2010;105:879-83.

6. Kim RJ, Wu E, Rafael A, et al. The use of contrast-enhanced magnetic resonance imaging to identify reversible myocardial dysfunction. N Engl J Med 2000;343:1445-53.

7. Moon JCC, Fisher NG, McKenna WJ, et al. Detection of apical hypertrophic cardiomyopathy by cardiovascular magnetic resonance in patients with nondiagnostic echocardiography. Heart 2004:90:645-9.

8. Tseng WY, Dou J, Reese TG, et al. Imaging myocardial fiber disarray and intramural strain hypokinesis in hypertrophic cardiomyopathy with MRI. J Magn Reson Imaging 2006;23:1-8.

9. Rickers C, Wilke NM, Jerosch-Herold M, et al. Utility of cardiac magnetic resonance imaging in the diagnosis of hypertrophic cardiomyopathy. Circulation 2005;112:855-61.

10. Matoh $\mathrm{F}$, Satoh $\mathrm{H}$, Shiraki $\mathrm{K}$, et al. Usefulness of delayed enhancement magnetic resonance imaging to differentiate dilated phase of hypertrophic cardiomyopathy and dilated cardiomyopathy. J Card Fail 2007;13:372-9.

11. Adabag AS, Maron BJ, Appelbaum E, et al. Occurrence and frequency of arrhythmias in hypertrophic cardiomyopathy in relation to delayed enhancement on cardiovascular magnetic resonance. J Am Coll Cardiol 2008;51:1369-74.

12. Rubinshtein R, Glockner JF, Ommen SR, et al. Characteristics and clinical significance of late gadolinium enhancement by contrast-enhanced magnetic resonance imaging in patients with hypertrophic cardiomyopathy. Circ Heart Fail 2010;3:51-8.

13. Fattori R, Biagini E, Lorenzini ML, et al. Significance of magnetic resonance imaging in apical hypertrophic cardiomyopathy. Am J Cardiol 2010;105:1592-6.

14. Sato T, Nakamura K, Yamanari H, et al. Relationship between electrocardiographic features and distribution of hypertrophy in patients with hypertrophic cardiomyopathy. Jpn Cric J 1998;62:483-8.

15. Satoh H, Matoh F, Shiraki K, et al. Delayed enhancement on cardiac magnetic resonance and clinical, morphological, and electrocardiographical features in hypertrophic cardiomyopathy. J Card Fail 2009;15:419-27.

16. Das MK, Khan B, Sony Jacob S, et al. Significance of a fragmented QRS complex versus a $Q$ wave in patients with coronary artery disease. Circulation 2006;113:2495-501.

17. Pietrasik G, Goldenberg I, Zdzienicka J, et al. Prognostic significance of fragmented QRS complex for predicting the risk of recurrent cardiac events in patients with $\mathrm{Q}$-wave myocardial infarction. Am J Cardiol 2007;100:583-6.

18. Natsume T, Amano T, Takehara Y, et al. Quantitative assessment of regional systolic and diastolic functions and temporal heterogeneity of myocardial contraction in patients with myocardial infarction using cine magnetic resonance imaging and Fourier fitting. Magn Reson Imaging 2009;27:1440-6.

19. Reddy V, Korcarz C, Weinert L, et al. Apical hypertrophic cardiomyopathy. Circulation 1998;98:2354

20. Ennezat PV, Mouquet F. Hypertrophic cardiomyopathy associated with left ventricular apical aneurysm. Arch Cardiovasc Dis 2010;103:198-9.

21. Matsubara K, Nakamura T, Kuribayashi T, et al. Sustained cavity obliteration and apical aneurysm formation in apical hypertrophic cardiomyopathy. J Am Coll Cardiol 2003;42:288-95.

22. Kubo T, Kitaoka H, Okawa M, et al. Clinical profiles of hypertrophic cardiomyopathy with apical phenotype-comparison of pure-apical form and distal-dominant form. Circ J 2009;73:2330-6.

23. Moon JCC, McKenna WJ, McCrohon JA, et al. Toward clinical risk assessment in hypertrophic cardiomyopathy with gadolinium cardiovascular magnetic resonance. J Am Coll Cardiol 2003;41:1561-7.

24. Kim RJ, Judd RM. Gadolinium-enhanced magnetic resonance imaging in hypertrophic cardiomyopathy: in vivo imaging of the pathologic substrate for premature cardiac death? J Am Coll Cardiol 2003:41:1568-72.

25. Dumont CA, Monserrat L, Soler R, et al. Interpretation of electrocardiographic abnormalities in hypertrophic cardiomyopathy with cardiac magnetic resonance. Eur Heart $J$ 2006;27:1725-31.

26. Koga $\mathrm{Y}$, Katoh A, Matsuyama K, et al. Disappearance of giant negative $T$ waves in patients with the Japanese form of apical hypertrophy. J Am Coll Cardiol 1995;26:1672-8.

27. Hata S, Shikuwa M, Yamasa T, et al. The left ventriculographic pattern and serial electrocardiographic changes in hypertrophic cardiomyopathy patients with giant negative T waves. Cardiology 1996;87:365-73.

28. Alfonso F, Nihoyannopoulos $\mathrm{P}$, Stewart J, et al. Clinical significance of giant negative $\mathrm{T}$ waves in hypertrophic cardiomyopathy. J Am Coll Cardiol 1990;15:965-71.

29. Chen X, Zhao T, Lu M, et al. The relationship between electrocardiographic changes and CMR features in asymptomatic or mildly symptomatic patients with hypertrophic cardiomyopathy. Int $J$ Cardiovasc Imaging 2014;30(Suppl 1):55-63.

30. Holloway CJ, Betts TR, Naubauer S, et al. Hypertrophic cardiomyopathy complicated by large apical aneurysm and thrombus, presenting as ventricular tachycardia. J Am Coll Cardiol 2010;56:1961. 